\title{
Progress in DGVMs: a comment on "Impacts of trait variation through observed trait-climate relationships on performance of an Earth system model: a conceptual analysis" by Verheijen et al. (2013)
}

\author{
S. I. Higgins ${ }^{1}$, L. Langan ${ }^{2}$, and S. Scheiter ${ }^{3}$ \\ ${ }^{1}$ Department of Botany, University of Otago, P.O. Box 56, Dunedin 9054, New Zealand \\ ${ }^{2}$ Institut für Physische Geographie, Goethe-Universität Frankfurt am Main, Frankfurt am Main, Germany \\ ${ }^{3}$ Biodiversität und Klima Forschungszentrum (LOEWE BiK-F), Senckenberg Gesellschaft für Naturforschung, \\ Frankfurt am Main, Germany \\ Correspondence to: S. I. Higgins (steven.higgins@otago.ac.nz)
}

Received: 5 February 2014 - Published in Biogeosciences Discuss.: 21 March 2014

Revised: 25 June 2014 - Accepted: 25 June 2014 - Published: 19 August 2014

Dynamic global vegetation models (DGVMs) are now central elements in Earth system models, and our ability to understand past and anticipate future changes in the Earth system is intimately linked to the quality of DGVMs (Prentice et al., 2007). There are many ways in which DGVMs need improvement and there are many exciting initiatives under way. In a recent manuscript, Verheijen et al. (2013) describe one pathway. To provide a context for their work, they compare their approach to other initiatives. In this contribution we wish to point out ways in which Verheijen and colleagues misrepresented the aDGVM2 (which they incorrectly call the aDGVM, which is in fact a different model published by Scheiter and Higgins, 2009) as presented in Scheiter et al. (2013). While the aim of this piece is primarily to set the record straight, we additionally point out similarities and differences between the approach described by Verheijen et al. (2013) and that described by Scheiter et al. (2013).

Verheijen et al. (2013) motivate their study by stating in reference to the Jena Diversity-DGVM (JeDi-DGVM) (Pavlick et al., 2013) and aDGVM2 (Scheiter et al., 2013) that "none of the approaches so far tried to maximally include trait variation based on observational trait data and capture multiple sources of this variation by relating trait data to environmental variables". Although we appreciate that this statement was designed to illustrate the uniqueness of Verheijen et al. (2013) and the statistical approach they adopt, it does have the side-effect of suggesting that these two papers ignored variations in traits and the relationships between traits and the environment. We would like to point out that Fig. 5 of our paper plots the positions of modelled individuals in multivariate trait space and relates the axes of this trait space to environmental variables.

In the same paragraph, the authors go on to suggest that DGVM modellers need to apply assembly theory to understand and model relationships between traits and the environment better, when they state that "such relationships between environmental conditions and traits can potentially be understood via ecological assembly theory". This is exactly what we propose in Scheiter et al. (2013), where the introduction explicitly proposes that DGVM modelling could benefit from two branches of community ecology, namely coexistence theory and community assembly theory. Moreover, the title of Scheiter et al. (2013) includes the words "learning from community ecology". Our impression from reading Pavlick et al. (2013) is that the traits that JeDi-DGVM predicts at a site are, as is the case with aDGVM2, a function of how environmental attributes select for trait combinations. This is an interpretation that Verheijen et al. (2013) appear, in apparent contradiction to their statement we cite above, to share in their discussion, when they state that "some DGVMs also implement the concept of environmental filtering, like the JeDi-DGVM (Pavlick et al., 2013)". 
In the discussion, JeDi-DGVM (Pavlick et al., 2013) is further criticised because its traits are "not-measurable". While we cannot assess what is measurable, we would like to point out that invitingly measurable traits are not inherently more useful than traits that can be inferred using inverse statistical methods (see Hartig et al., 2012, for an overview of using inverse methods in the context of DGVMs).

At a prominent point in the discussion, Verheijen states that the "aDGVM has not been validated with observational data". This statement serves as invitation to the reader to ignore the aDGVM2. This is a curious criticism of our work, because we never claimed the aDGVM2 to be validated; the paper in question was explicitly a method and concept paper, and we did not make any forecasts. Furthermore, we are sure that most authors of DGVMs would not claim to have authored validated models. At best, a DGVM can claim to have passed some benchmarks or to provide a better benchmark score than competitor models. Furthermore, should an author pronounce a model to be "validated", this pronouncement would not be universal, but would be restricted to the domain of that study. This criticism is even more curious considering that the authors themselves, at the conclusion of the introduction, state that their study does not aim to produce "realistic results" and that their focus lies in "evaluating the importance of incorporating climate-driven trait variation". This disclaimer seems ad hoc, given that considerable space in the manuscript is devoted to benchmarking the model and explaining why the benchmarks used might undervalue the performance of their modelling approach (e.g. "our simulations with 7 vegetation classes performed less well, but this might partly depend on the chosen vegetation map" and "this implies that the estimates of GPP by Beer et al. (2010) might be too low"), space that could have been used for "evaluating the importance of incorporating climate-driven trait variation". Perhaps the disclaimer was added because the new parameterisation method developed in the Verheijen paper yielded substantially poorer benchmark scores than the existing parameterisation method. The different performances of the parameterisation methods support our contention (Scheiter et al., 2013) that hidden calibration in DGVMs inflates their capacity to match benchmarks and hides model misspecification, something that objective parameterisation schemes will reveal. The Verheijen manuscript, which essentially compares a hidden calibration parameterisation method with two objective parameterisation methods, illustrates this point nicely.

To be more constructive, we would like to point out important differences between our approach and that adopted by Verheijen et al. (2013). We explicitly chose not to follow the approach used by Verheijen et al. (2013), which was to use direct statistical inference to parameterise plant trait diversity. The pragmatic but inherent problem with this approach is that there is no $1: 1$ match between the parameters in trait databases and the parameters DGVMs use. In fact, it appears, on the evidence of the Verheijen manuscript, that only three JSBACH (the DGVM used in the Verheijen study) traits matched traits in TRY (Kattge et al., 2011) and other trait databases used in Verheijen et al. (2013). Our approach attempts to side-step the parameter incongruence problem by instead focusing on defining trade-offs between traits. Verheijen et al. (2013) correctly point out in their discussion that model architecture constrains how trade-offs are represented, and they identify cases where JSBACH is, in this regard, limited. Verheijen et al. (2013) then state that "aDGVM has not been validated with observational data nor does it include trait trade-offs". This second strongly dismissive statement is perplexing, given that the central tenet of the aDGVM2 is to focus not so much on the traits, but on the trade-offs between traits. In Scheiter et al. (2013), a paper cited by the authors, we identified trade-offs and their representation in DGVMs as the central challenge for next-generation DGVMs. We discuss at some length how to implement such trade-offs in DGVMs and we used the aDGVM2 to illustrate some of these ideas. Some trade-offs in the aDGVM2 are empirically defined, but others are emergent consequences of conservation of mass principles and mechanical constraints implemented in the model (Scheiter et al., 2013). Once trade-offs are defined, the actual trait values a plant may adopt in an aDGVM2 simulation are the outcome of trait filtering. We use the term "trait filtering" as a shorthand for how the ecological processes implemented in the model define the trait combinations that persist in a simulation (Figs. 2 and 4 in Scheiter et al., 2013). A constructive criticism of Scheiter et al. (2013) would involve pointing out whether important trade-offs are missing from the aDGVM2 or whether the trade-offs included are misspecified.

A further difference is that Verheijen and colleagues use statistical smoothing methods to estimate how the three traits that are congruent between the JSBACH model and the trait databases vary in environmental space, and then use the resulting functions as a lookup table to reparameterise every simulation year for each grid cell and the traits of each plant functional type. In this context they criticise aDGVM2, stating that in the aDGVM2, "environmental filtering only acts on trait values through the next generation". This is not entirely correct. The aDGVM2 allows thousands of individual plants, each with their own potentially unique set of traits, to exist in a simulated vegetation patch. These individuals can die at any modelled time step, as influenced by disturbance, competition and resource availability. In addition, other individuals with potentially novel trait combinations can germinate each year. As a consequence of these birth and death processes, the community trait matrix will change every modelled time step. Furthermore, in the aDGVM2, we make a distinction between traits (inherited attributes) and phenotypes (the outcome of interactions between an organism's traits and its environment). Traits of a modelled individual cannot vary in the lifetime of that individual, but a modelled individual's phenotype can change as it grows and is subjected to disturbance. In summary, the phenotypes 
of individuals are modelled to change each simulation time step, and the community trait matrix changes every time an individual is born and every time an individual dies. It follows that the criticism that the aDGVM2 approach is flawed because it does not allow "traits" to vary between years is distracting and without substance.

A related problem with using statistical methods to parameterise functional diversity in DGVMs is that the dimensionality of the parameterisation task is high. The Verheijen et al. (2013) approach essentially requires a different parameterisation for each time step, for each geographic location, and for each plant functional type. Even though using correlations between environmental factors and traits is done to reduce the dimensionality of the task, many parameters are needed. A side-effect of this large number of parameters is that it provides the modeller with the flexibility to tune the model to a benchmark. As we discuss in Scheiter et al. (2013), one advantage of our method of combing trade-offs and trait filtering is that the dimensionality of the functional diversity parameterisation does not change with the number of functional types or with the number of geographical locations simulated.

A further reason why we would not advocate Verheijen et al.'s (2013) statistical route to describing trait variation is that it is well known that within a plant functional type at a site there is a large range of trait states, simply because species with differing trait values are more likely to coexist (MacArthur and Levins, 1967). Cody's (1986) delightful example of the divergent leaf traits of coexisting species of Proteaceae shrubs illustrates this point nicely. It follows that additional information and assumptions regarding limitations on the similarity of species need to be made when developing statistical models of community assembly (Laughlin and Laughlin, 2013). Future community assembly will be conditional on each locality's community trait matrix, and the community matrix is in turn defined, in part, by history: that is, while a statistical approach seems pragmatic, it is not clear whether identifiable statistical models and appropriate data that describe all the important sources of variation can be defined. By aggregating the effects of "different temporal and spatial scales, including acclimation, adaptation of species and species replacement" in statistical models (with a median $R^{2}$ value of 0.36 ), Verheijen et al. (2013) actually smooth away substantial components of the variation they themselves recognise as being essential for next-generation DGVMs.

We readily concede that the aDGVM2 as published in Scheiter et al. (2013) is a starting point, an illustration of the promise of one approach, and a vehicle for encouraging more intimate interaction between trait data and DGVMs. We are disappointed that Verheijen and colleagues felt it necessary to dismiss our contribution. To criticise a concept model because it is "not-validated" misses the point of a concept model. To criticise a published work for not "relating trait data to environmental variables" or for "not including trade- offs" when that work quite transparently does both is a questionable way to make progress in science.

We hope that this comment has made some of the differences between the two approaches more apparent to both developers and users of DGVMs. In summary, the important difference is that Verheijen et al. (2013) use a direct statistical method to parameterise plant functional diversity, whereas Scheiter et al. (2013) and Pavlick et al. (2013) define trade-offs between plant functional traits, which allow functional diversity to emerge as a by-product of model dynamics. The approach followed by Scheiter et al. (2013) and Pavlick et al. (2013) is reliant on the specification of tradeoffs between functional traits and how they together with modelled and forced environmental factors influence birth, death and growth rates in the models. While this may sound simple in principle, Scheiter et al. (2013) outline some of the non-trivial challenges involved in developing such models to the stage where they can approach the reliability of existing DGVMs.

Acknowledgements. Financial support was provided by the Deutsche Forschungsgemeinschaft (DFG) and Hesse's Landesoffensive zur Entwicklung wissenschaftlich-Ökonomischer Exzellenz (LOEWE).

Edited by: S. Zaehle

\section{References}

Cody, M. L.: Structural niches in plant communities, in: Community Ecology, edited by: Diamond, J. M., Harper and Row, New York, NY, 381-405, 1986.

Hartig, F., Dyke, J., Hickler, T., Higgins, S. I., O'Hara, R. B., Scheiter, S., and Huth, A.: Connecting dynamic vegetation models to data - an inverse perspective, J. Biogeogr., 39, 2240-2252, 2012.

Kattge, J., Díaz, S., Lavorel, S., Prentice, I. C., Leadley, P., Bönisch, G., Garnier, E., Westoby, M., Reich, P. B., Wright, I. J., Cornelissen, J. H. C., Violle, C., Harrison, S. P., Van Bodegom, P. M., Reichstein, M., Enquist, B. J., Soudzilovskaia, N. A., Ackerly, D. D., Anand, M., Atkin, O., Bahn, M., Baker, T. R., Baldocchi, D., Bekker, R., Blanco, C. C., Blonder, B., Bond, W. J., Bradstock, R., Bunker, D. E., Casanoves, F., Cavender-Bares, J., Chambers, J. Q., Chapin III, F. S., Chave, J., Coomes, D., Cornwell, W. K., Craine, J. M., Dobrin, B. H., Duarte, L., Durka, W., Elser, J., Esser, G., Estiarte, M., Fagan, W. F., Fang, J., Fernández-Méndez, F., Fidelis, A., Finegan, B., Flores, O., Ford, H., Frank, D., Freschet, G. T., Fyllas, N. M., Gallagher, R. V., Green, W. A., Gutierrez, A. G., Hickler, T., Higgins, S. I., Hodgson, J. G., Jalili, A., Jansen, S., Joly, C. A., Kerkhoff, A. J., Kirkup, D., Kitajima, K., Kleyer, M., Klotz, S., Knops, J. M. H., Kramer, K., Kühn, I., Kurokawa, H., Laughlin, D., Lee, T. D., Leishman, M., Lens, F., Lenz, T., Lewis, S. L., Lloyd, J., Llusià, J., Louault, F., Ma, S., Mahecha, M. D., Manning, P., Massad, T., Medlyn, B. E., Messier, J., Moles, A. T., Müller, S. C., Nadrowski, K., 
Naeem, S., Niinemets, Ã., Nöllert, S., Nüske, A., Ogaya, R., Oleksyn, J., Onipchenko, V. G., Onoda, Y., Ordoñez, J., Overbeck, G., Ozinga, W. A., Patiño, S., Paula, S., Pausas, J. G., Peñuelas, J., Phillips, O. L., Pillar, V., Poorter, H., Poorter, L., Poschlod, P., Prinzing, A., Proulx, R., Rammig, A., Reinsch, S., Reu, B., Sack, L., Salgado-Negret, B., Sardans, J., Shiodera, S., Shipley, B., Siefert, A., Sosinski, E., Soussana, J.-F., Swaine, E., Swenson, N., Thompson, K., Thornton, P., Waldram, M., Weiher, E., White, M., White, S., Wright, S. J., Yguel, B., Zaehle, S., Zanne, A. E., and Wirth, C.: TRY - a global database of plant traits, Glob. Change Biol., 17, 2905-2935, 2011.

Laughlin, D. C. and Laughlin, D. E.: Advances in modeling traitbased plant community assembly, Trends Plant Sci., 18, 584593, 2013.

MacArthur, R. and Levins, R.: The limiting similarity, convergence, and divergence of coexisting species, Am. Nat., 101, 377-385, 1967.

Pavlick, R., Drewry, D. T., Bohn, K., Reu, B., and Kleidon, A.: The Jena Diversity-Dynamic Global Vegetation Model (JeDiDGVM): a diverse approach to representing terrestrial biogeography and biogeochemistry based on plant functional trade-offs, Biogeosciences, 10, 4137-4177, doi:10.5194/bg-10-4137-2013, 2013.
Prentice, I. C., Bondeau, A., Cramer, W., Harrison, S. P., Hickler, T., Lucht, W., Sitch, S., Smith, B., and Sykes, M. T.: Dynamic Global Vegetation Modeling: quantifying terrestrial ecosystem responses to large-scale environmental change, in: Terrestrial Ecosystems in a Changing World, edited by: Canadell, J. G., Pataki, D. E., and Pitelka, L. F., Global Change - The IGBP Series, Springer, Berlin Heidelberg, 175-192, 2007.

Scheiter, S. and Higgins, S. I.: Impacts of climate change on the vegetation of Africa: an adaptive dynamic vegetation modelling approach, Glob. Change Biol., 15, 2224-2246, 2009.

Scheiter, S., Langan, L., and Higgins, S. I.: Next-generation dynamic global vegetation models: learning from community ecology, New Phytol., 198, 957-969, 2013.

Verheijen, L. M., Brovkin, V., Aerts, R., Bönisch, G., Cornelissen, J. H. C., Kattge, J., Reich, P. B., Wright, I. J., and van Bodegom, P. M.: Impacts of trait variation through observed trait-climate relationships on performance of an Earth system model: a conceptual analysis, Biogeosciences, 10, 5497-5515, doi:10.5194/bg-10-5497-2013, 2013. 